\title{
Early Augmentation Response with Low-frequency Repetitive Transcranial Magnetic Stimulation in Treatment Resistant Depression
}

\author{
Jyoti Singh, Amit Singh, Sujita Kumar Kar, Erika Pahuja \\ Department of Psychiatry, King George's Medical University, Lucknow, Uttar Pradesh, India
}

\section{TO THE EDITOR}

Repetitive transcranial magnetic stimulation (rTMS) has been used for management of treatment resistant depression over few decades. Evidences suggest the use of high-frequency $(\geq 10 \mathrm{~Hz})$ rTMS over left dorsolateral prefrontal cortex (DLPFC) and low-frequency rTMS over right DLPFC. ${ }^{1)}$ It has been approved by the US Food and Drug Administration in 2008 for treatment of depression. ${ }^{2)}$ Recent meta-analysis suggests about the similar efficacy of left and right sided rTMS in depression, though the later is less studied. ${ }^{3)}$ We highlight the efficacy and some unique response characteristics of low-frequency rTMS in our patient.

A 50 year-old non-diabetic, non-hypertensive male presented with complaints of low mood, anhedonia, anxiety, difficulty in concentration, reduced sleep and appetite for 18 months. These symptoms were causing significant socio-occupational impairment. For these complaints, he was treated with various antidepressants (escitalopram, bupropion, paroxetine, vilazodone, and mirtazapine) in adequate doses alone and in combination for adequate period of time during the initial part of this episode prior to hospitalization in our center. Due to poor response to treatment, augmentation had also been done with alpha-methyl-folate, risperidone and modafinil in past. His routine hemogram, liver and renal function tests, thyroid function test and neuro-imaging were within normal limits.

His past and family history was non-contributory. He was diagnosed with major depressive disorder and prescribed sertraline at a dose of $50 \mathrm{mg}$ /day. Due to non-response to this treatment in 4 weeks, he was hospitalized

\footnotetext{
Received: March 2, 2016 / Revised: April 19, 2016

Accepted: April 20, 2016

Address for correspondence: Sujita Kumar Kar, MD

Department of Psychiatry, King George's Medical University, Shah

Mina Road, Chowk, Lucknow, Uttar Pradesh 226003, India

Tel: +91-522-2258881, Fax: +91-522-2258805

E-mail: skkar1981@yahoo.com
}

and rTMS augmentation was done after obtaining informed consent. The resting motor threshold (RMT) was determined using the figure 8 coil and low-frequency rTMS was delivered over right DLPFC. The TMS protocol used was 20 trains of $1 \mathrm{~Hz}$, with 60 pulses/trains and an inter-train interval of 5 seconds ( 1,200 pulses/session) for 18 sessions at $100 \%$ of RMT over three weeks. The baseline score on Montgomery-Åsberg Depression Rating Scale (MADRS) was 26, which was reduced to 10 after 12 sessions and 6 by the end of therapy. The patient was maintained well at 8 weeks follow up. The patient also had nicotine dependence which was successfully treated with nicotine replacement therapy.

In our patient, we had used the TMS protocol with 1,200 pulses/session over 18 sessions (total 21,600 pulses). A recent meta-analysis suggests that use of $>1,200$ pulses in therapy to be more effective. ${ }^{4)}$ The therapeutic response was observable as early as 12th session (2nd week), as evident by the reduction in MADRS score and it sustained over next 8 weeks. In our case, TMS was effective in augmenting the antidepressant effect of $50 \mathrm{mg} /$ day sertraline. The unique features in this case were early response to rTMS augmentation and elicitation of augmentation response on relatively lower dose of antidepressant. It can be certainly said that despite of multiple poor prognostic factors (50 years of age, 18 months long depressive episode, treatment resistance), rTMS can augment the antidepressant effect. The above TMS protocol may be used as an augmentation strategy for treatment-resistant depression and TMS augmentation can even be started with low dose concomitant antidepressant treatment.

\section{REFERENCES}

1. Lefaucheur JP, André-Obadia N, Antal A, Ayache SS, Baeken $\mathrm{C}$, Benninger DH, et al. Evidence-based guidelines on the therapeutic use of repetitive transcranial magnetic stimulation (rTMS). Clin Neurophysiol 2014;125:2150-2206.

2. Aleman A. Use of repetitive transcranial magnetic stimulation for treatment in psychiatry. Clin Psychopharmacol Neurosci

(a) This is an Open-Access article distributed under the terms of the Creative Commons Attribution Non-Commercial License (http://creativecommons.org/licenses/by-nc/4.0) which permits unrestricted non-commercial use, distribution, and reproduction in any medium, provided the original work is properly cited. 
2013;11:53-59.

3. Chen J, Zhou C, Wu B, Wang Y, Li Q, Wei Y, et al. Left versus right repetitive transcranial magnetic stimulation in treating major depression: a meta-analysis of randomised controlled trials. Psychiatry Res 2013;210:1260-1264.

4. Berlim MT, Van den Eynde F, Jeff Daskalakis Z. Clinically meaningful efficacy and acceptability of low-frequency repetitive transcranial magnetic stimulation (rTMS) for treating primary major depression: a meta-analysis of randomized, double-blind and sham-controlled trials. Neuropsychopharmacology 2013;38:543-551. 